\title{
Interprofessional Simulation Learning with Nursing and Pharmacy Students: A Qualitative Study
}

\author{
Pauline Paul \\ Faculty of Nursing, University of Alberta, ppaul@ualberta.ca \\ Joanne K. Olson \\ University of Alberta, joanne.olson@ualberta.ca \\ Cheryl Sadowski \\ cheryl.sadowski@ualberta.ca \\ Brian Parker \\ ParkerB5@mcewan.ca \\ Angele Alook \\ angele.alook@ualberta.ca \\ Deirdre Jackman \\ deirdre.jackman@ualberta.ca \\ Cheryl Cox \\ cheryl.cox@ualberta.ca \\ Stewart MacLennan \\ stewart.maclennan@ualberta.ca
}

Follow this and additional works at: https://qane-afi.casn.ca/journal

Part of the Nursing Commons, and the Pharmacy and Pharmaceutical Sciences Commons

\section{Recommended Citation}

Paul, Pauline; Olson, Joanne K.; Sadowski, Cheryl; Parker, Brian; Alook, Angele; Jackman, Deirdre; Cox, Cheryl; and MacLennan, Stewart (2014) "Interprofessional Simulation Learning with Nursing and Pharmacy Students: A Qualitative Study," Quality Advancement in Nursing Education - Avancées en formation infirmière: Vol. 1: Iss. 1, Article 6.

DOI: https://doi.org/10.17483/2368-6669.1011

This Article is brought to you for free and open access by Quality Advancement in Nursing Education - Avancées en formation infirmière. It has been accepted for inclusion in Quality Advancement in Nursing Education - Avancées en formation infirmière by an authorized editor of Quality Advancement in Nursing Education - Avancées en formation infirmière. 


\section{Interprofessional Simulation Learning with Nursing and Pharmacy Students: A}

Qualitative Study

\section{Cover Page Footnote}

This research was funded by the Faculty of Nursing, University of Alberta. The authors thank Nadine Moniz, Darren Pasey, Kerry Rusk, and Karen Sylte for their assistance with this project. 
Health science students are increasingly exposed to simulation learning to promote disciplinespecific education. However, uni-professional simulation learning is no longer sufficient. Future health care practitioners need opportunities in their education to practice interprofessional collaboration skills. Registered nurses and pharmacists are two groups of health professionals that must learn to work together, as their collaboration is necessary for the provision of optimal patient care. Furthermore, organizations that approve and accredit health sciences programs have increasing expectations with respect to interprofessional learning opportunities to meet competencies for interprofessional collaboration (Bainbridge, Nansmith, Orchard, \& Wood, 2010). The purpose of this study, which occurred in a large Canadian university, was to seek perceptions of undergraduate nursing and pharmacy students on how interprofessional simulation learning (ISL) facilitates the development of disciplinary learning as well as interprofessional skills.

\section{Background}

ISL can contribute to increasing students' understanding of professional roles and team building in clinical settings (Baker et al., 2008; Dagnone, McGraw, Pulling, \& Patterson, 2008; Fernandez, Parker, Kalus, Miller, \& Compton, 2007; Gum, Greenhill, \& Dix, 2010; MacDonnell, Derresa, Lavin, Cohen, \& Cohen, 2011; O’Neill \& Wyness, 2005). ISL has the potential to decrease errors, improve communication, and improve teamwork (Merién, van de Ven, Mol, Houterman, \& Oei, 2010). Studies with pharmacy and nursing students learning together suggest increased collaboration, comfort, confidence, and clinical knowledge after ISL experiences (Efstathiou \& Walker, 2013; MacDonnell et al., 2011; O’Neill \& Wyness, 2005; Westberg et al., 2006).

Health Canada (2006) highlights the need for continued research related to interprofessional education. Robertson and Bandali (2008) called for research to build a body of evidence to guide educators in designing curricula using ISL. Research examining the combination of simulation and interprofessional learning is especially needed in the Canadian context since few studies have been conducted in this country (Bandali, Parker, Mummery, \& Preece, 2008). The specific research question in this study was: What are the perceptions of nursing and pharmacy students regarding the ISL experience designed for this study, in terms of: a) discipline specific learning, b) interprofessional learning, c) understanding of their own professional roles, d) understanding the roles of their colleagues, and e) collaborative decision making?

\section{Methods}

This exploratory descriptive qualitative study used an ethnographic approach (Field \& Morse, 1985), which provides insights into "the context, the people, and the interactions of practice" (Roper \& Shapira, 2000, p. 25). This approach allows for increased understanding of the student experience when two professional cultures converged. Nursing and pharmacy students enrolled in the final year of their undergraduate programs were invited to participate via email once institutional ethical clearance had been granted.

All participants were exposed to three simulation learning activities over six hours: one nursing student-led high fidelity simulation; one pharmacy student-led standardized patient simulation; 
and one collaborative interprofessional standardized patient simulation. High fidelity simulation uses a technologically advanced patient model to produce physical and verbal signals that lead the learner to critically reflect and care for an acutely ill patient. A standardized patient simulation uses an actor who plays the role of a patient experiencing a health situation. Simulations were based on existing scenarios used in pharmacy and nursing curricula. The first two simulations used scenarios located in acute care contexts and gave participants the opportunity to observe students from the other discipline. The third simulation used a scenario located in a primary care setting and was designed to promote joint participation.

All research team members were involved in the simulation activities and data collection, which included observation of simulations and debriefing sessions, individual interviews, and field notes. Five research team members conducted individual interviews using the same interview guide. Data collection occurred throughout January and February 2013. All interviews occurred within two weeks after the simulation experiences to maximize participant recall of the interprofessional interactions.

Data analysis was conducted by all team members using content analysis (Graneheim \& Lundman, 2003; Vaismoradi, Turunen, \& Bondas, 2013). Codes were generated from the data and then organized into categories, and meaningful themes were generated. Observation data and researcher field notes were examined and included as part of the analysis. Issues of rigor were addressed in relation to credibility, dependability, confirmability, trustworthiness, and transferability.

\section{Results}

\section{Sample}

Participants included five nursing students and four pharmacy students in the final year of their programs. All of the nursing students were female; while all but one of the pharmacy students were male. Participant numbers were sufficient to provide rich descriptive data. Findings were reported to answer the research questions using the following headings: general impression of ISL experience, understanding their own professional roles, understanding the roles of colleagues from another profession, and learning more about interprofessional collaboration.

\section{General impressions of ISL experience}

Student participants found the ISL activities to be positive learning experiences. They described themselves as comfortable and self-confident. As one nursing student expressed:

"I found this simulation to be my most enjoyable ... I've found it to be appropriately comfortable for learning".

Being observed by other students was generally not considered threatening; rather, working with students from a different discipline appeared to reduce the inherent stress of most simulated learning sessions. As noted by one student:

"It was surprisingly easy just to feel comfortable and feel at ease with each other". 
The social aspect of bonding and building relationships was important to the experience and participants described taking breaks with students from another discipline as a memorable aspect. One pharmacy student cited the "down time" and the ability to "banter" as positive aspects:

"I felt comfortable with my peers from the nursing faculty".

The activities were described as fun and enjoyable. Another nursing student described the value of social connections with peers for building relationships:

"I think just being able to talk to the pharmacy students and even the moments in between simulations when we were in a break, we just sat and talked and asked each other questions 'what would you do, how would you approach this?' And that was really neat to just ask questions."

\section{Understanding their own professional roles}

Both professions showed pride in their ability to build rapport with patients during the ISL activities. Nursing students mentioned how they see patients throughout the day and come to know them quite intimately. As one nursing student stated:

"Our job is so invasive [intimate] so we get used to it ... to how you should talk with a client ... whereas in pharmacy, their role is entirely different, they're not providing the invasive peri-care ... so I think as nurses we get used to one-on-one interactions."

Another aspect of building rapport with patients noted by nursing students was their ability to get to know patients and their families personally:

"I think just our knowledge about the patient, knowing who they are as a person and just being comfortable around them".

Pharmacy students indicated that they might have fewer patients throughout the day in a hospital setting and therefore ample time to talk with each. One pharmacy student recognized that "... physicians and nurses do have a lot on their plate" and the pharmacists are normally "not actually assigned to a particular patient". He went on to say that having more time with patients allows the pharmacist to "develop that rapport and find out more about the patient ... that other health care team members don't know and we can provide them with that information". Overall, both nursing and pharmacy students mentioned how building rapport with patients is an important aspect of their professional role that allows them to be an asset to a healthcare team. All participants stated that this activity created pride in their own profession; however connecting with a different profession in a personal and professional sense also set the tone for a collaborative work environment. One nursing student mentioned that building rapport with colleagues was as important as building rapport with patients when providing care:

"I like to find out someone's name and how they are doing today before I even say 'okay (Physical Therapist) what do you have to say about this patient' ... I think it makes the workplace a little friendlier and we can't all just be clinicians all the time." 
Nursing students mentioned how providing holistic care and patient safety are important for patient-centred care. One nursing student illuminated the various skills and knowledge an $\mathrm{RN}$ uses when providing patient care and said the ISL activities confirmed the importance of the holistic aspect of care:

"We are expected to know those side effects and metabolism and absorption and excretion of medications ... and then there is the rapport building and the concerns about a family's financial situation and there's the co-morbidity and ... the pathophysiology."

For pharmacy students, the activity highlighted their professional clinical role related to providing patient-centred care on an interprofessional team. One pharmacy student thought "pharmacists have a big role to play" and this was made clear during the scenario that required "managing the person with diabetes ... we do know a lot, and we can work with them [nursing students/RNs]".

Each profession has a specific knowledge base that contributes to an interdisciplinary team. One nursing student mentioned that, "when you are with another discipline, you think 'okay I know a little bit about this particular situation' that makes my skills and knowledge valuable and it's not the same as pharmacists". Another mentioned that nurses have "special roles" and "something to offer to the team". During the simulation, she noticed the pharmacy student and thought "okay, I have some things I can help you with ... I could let them know we could move the patient if they need to be made more comfortable (during the meeting with the pharmacist)". For pharmacy students, the ISL highlighted their role as specialists with in-depth medication knowledge. As "a drug information specialist", one pharmacy student noted they "got asked a lot of questions about the medications" and, through discussion, were able to notice that the antibiotic was incompatible with the solution. One student noted the importance of medication knowledge during critical periods:

"Pharmacy isn't all just about looking things up in a book. It reminded me that we need to know information off the top of our heads. There are times when we are involved in those quick calls and ... it really opened my eyes that we are a very important part of the interdisciplinary team."

\section{Understanding the roles of colleagues from another profession}

Participants discussed similarities between their professional cultures and how their roles may overlap. For example, a nursing student discussed how she was impressed when observing a pharmacy student interact with a patient: "I just hadn't considered that a pharmacist would take a past medication history". The student expected this 'medication historian' role to involve a physician or an $\mathrm{RN}$, and was interested to learn that pharmacists also develop and value this role. Commenting on an assessment by a pharmacy student, a nursing student elaborated on the different scopes of practice but similar roles:

"I felt that she [the pharmacy student] did a really good assessment ... she also thinks of the head to toe assessment which is very similar to what we think of in nursing ... it's just different because they do it all at once in one meeting while we are doing ongoing assessments." 
Nursing students were surprised to realize that pharmacists also valued patient-centred care as a cornerstone of their practice. One appreciated how a pharmacy student considered patient priorities in their thinking and decision making during consultation:

"I see that's what's important to you [patient], it's not continuously pricking your finger, so let's come up with an alternative, even though my priorities are that you just need to get it done, you need to be checking your blood sugars, but we have to factor in [your] priorities ..."

In return, pharmacy students noted the superb communication skills nursing students had when talking with patients, and how these skills led to in-depth patient and family knowledge. As one pharmacy student remarked:

"So they know more about the patient's condition, for example how they were sleeping, how they are doing with their medications ... And talking with the patient I guess, because what I've seen during the labs, two students that I was with were really, really great in talking with the patient, they have a lot of empathy. I remember in first year in communications classes, our professor told us empathy is really important especially when you are talking with patients ... it's really difficult to actually connect with the patient, but I've seen two nursing students in that lab who have done it really well."

Pharmacy students learned they could use nursing colleagues as resources in future practice to better understand specific patient circumstances and to improve care. As one pharmacy student commented:

"We are all geared to patient-centred care, all there to assess the patient, using our best assessment and our best judgement of those patients to provide the [best] patient care that we can."

Many stereotypical perceptions dissipated as students learned more about each other. For example, the perception that nurses value teamwork and pharmacists value working independently was challenged. One pharmacy student stated that:

“... [for nursing students] there was a lot of team work going on, and they told us they do require that kind of team work whereas pharmacy students are encouraged within their education program to become independent like physicians, having an independent way of thinking ... we make our own decisions ... but I think teamwork is also important."

One nursing student initially described pharmacists as "scientists, thrown into social situations, which may not be their strength". However, after the ISL activity, they recognized that pharmacists need to develop strong communication skills to interact with patients. The activity also challenged students to reflect on how each profession requires both teamwork and autonomy; as they spent time together and socialized, they learned that previously held stereotypes did not align with their ISL experiences of each other's actual professional role(s).

Students recognized the importance of interacting in order to build both social and professional relationships. As noted by one nursing student: 
"Of course there are differences, but the similarities are ... that we all want to do what's best for the client ... . Before I was aware of the different scopes [of practice] and what the pharmacist does in comparison to nursing, but it was actually really cool to realise how similar we are in a lot of ways, so we are a team."

The shared purpose was specifically noted by another nursing student: "we all had the same goal of getting the patient out of the hospital and back home ... the goals are the same even if the routes to getting there are somewhat different". The importance of the team approach was also explicitly recognized by one pharmacy student:

"The future of health care is teamwork, so being able to work with one another, our scopes of practice mesh together. There are [situations] that are different, but we do have to rely on other professionals' expertise in order to give the patient the best outcome."

\section{Learning interprofessional collaboration}

Several students noted that they were more comfortable with another profession after the ISL activities. One nursing student felt the simulation activities helped her collaborate, emphasizing the "need to communicate" in order to "keep ... patients safe" and "get stuff done as a team". One pharmacy student learned that effective collaboration is to "choose words wisely, so that there is no communication breakdown". He recognized that different disciplines have different perspectives and need to ensure that "everyone is on the same playing field with using the same terminology". As one nursing participant put it, she was just "more open to collaborating" after the ISL activities because she realized; "I can't do everything for a patient, so I want to be able to call on people who can do the right thing when I can't".

The ISL activities increased participant comfort with respect to employing the other profession as a resource. Many participants discussed how a nurse could help the pharmacist build rapport with the patient and how the pharmacist could be readily called upon in a clinical environment for a medication consultation. One nursing participant noted a particularly valuable lesson was "the importance of not thinking of your profession as the only profession, [and] actually seeking other health care providers for their help in specific situations that would be appropriate". Another nursing student felt she could consult other professions in future practice:

"I'll certainly feel more comfortable going to a pharmacist in the hospital or even in the community and asking them for information or specific things about their practice that I wouldn't have thought to do before".

A strong learning outcome of the ISL activities was the recognition by both groups of students that interprofessional collaboration and teamwork are valued by each of their professions. This involves recognizing each other as part of a team and being willing to use each other as resources. For example, one nursing student discussed having a pharmacist as a resource:

“... we can just call them up ... can we put these medications with these solutions? So just knowing they are available is really helpful ... . I felt more confident we were giving the patient good care, I also felt more confident myself." 
In addition, pharmacy students were willing to be a resource to their colleagues when their expertise is needed:

"We were able to collaborate very well with other professional health care members, especially with the nurses in their second simulation; they sort of referred to us regarding our drug management skills and sort of learned how important pharmacists can actually be in a hospital setting."

Both nursing and pharmacy students learned that good healthcare requires teamwork. A pharmacy student discussed the importance of thinking outside professional boundaries:

"Sometimes staying in your own profession is great and everything, but you really sort of need to reach outwards and see what other professions have to offer, because only if you do that can you truly use the entire knowledge base of the other profession and provide the best patient care."

One nursing student admitted that:

"Sometimes it just feels like we're all just nurses and we are part of our own nursing teams and our nursing culture ... and I still very much see it with nursing glasses on".

However, she remarked on how meeting two pharmacy students in the ISL experience made her feel more comfortable talking to someone outside her discipline and more open to consulting with other healthcare professionals.

In order to work well on a team, one pharmacy student commented on the "need to have a relationship" in order to collaborate with others, highlighting the "need to know these people, have a feel for what they're doing, [and] what their goals are with patients". Some of the participants suggested that this means getting to know colleagues on a healthcare team on a professional level in terms of their expertise but also on a personal level as human beings caring for others. One nursing student recognized "a connection of humanity" in the two pharmacy students she worked with, remarking that "it might not be the same with everybody, but it's a hugely important quality when you are working in these kinds of areas".

\section{Discussion}

The thematic analysis of the findings is reflected in the interrelated theoretical constructs of social learning theory and Mezirow's (1994, 1995) transformative learning theory. Modern health education is challenged with not only socializing students into individual professions but also helping them develop knowledge and skills applicable to working in an interprofessional environment. Mezirow's theory focuses on the role of experience, social discourse, and critical reflection in transforming a learner's 'meaning' regarding their experiences within a particular situation, in this case ISL. Here, the ISL challenged students' knowledge, values, and beliefs about clinical practice and other health disciplines. This in turn helped them to ultimately incorporate the new meanings into their cognitive schema. Similar to constructivist pedagogy, transformative learning theory promotes educational practices to empower learners. 
The related field of social learning theory also provides a theoretical construct on which to frame the findings from this study. Key aspects of social learning theory linked to the findings include the belief that learning is a social activity (Durning \& Artino, 2011; Taylor \& Hamdy, 2013). This form of social activity requires group dialogue and peer filtering of knowledge in order to transform meaning and promote collaboration (Parker \& Myrick, 2010; Tinsley \& Lebak, 2009). In addition, for quality learning to occur, educators must consider the key factors of context and community (Durning \& Artino, 2011). The ISL environment provides students with a community of social learning in a context (i.e., healthcare scenario) that promotes the benefits of peer-filtering of knowledge and social dialogue reflective of the realities in the modern healthcare setting. The feedback individual learners gain from peers serves to enhance not only their understanding of each other's disciplines, but also their skills with critical reflexivity (Tinsley \& Lebak, 2009).

Wells (2000) described the benefits of social learning with peers, noting that collaboration enhances knowledge, especially when learners are responsive to the contributions of others. This responsiveness also enlightens the learner to the meanings, values, and beliefs behind their own responses. This enlightenment facilitated through the ISL scenarios ultimately served to build a sense of community amongst the participants. There is a commonality between professional cultures (Hean, Clark, Adam, \& Humphris, 2006), but stereotypical perceptions can emerge when different health professional cultures encounter each other (Ateah, Snow, Wener et al., 2011). The ISL activities helped students learn about and gain pride in their own profession while also learning to appreciate another profession and overcome these stereotypes. The study also demonstrates the value of ISL activities for providing students with opportunities for a high level of engagement in interprofessional learning. These findings echo the theoretical foundations that anchored this study as well as support the promise of interprofessional education for improving the knowledge, skills, confidence, and competence of students (Efsathkiou \& Walker, 2013) and preparing them for collaborative models of healthcare delivery (Baker et al., 2008).

A unique finding of the study is the extent to which students found the ISL provided an opportunity for building both professional and personal relationships; this was as important as the actual simulation learning. These opportunities for relationship building occurred mainly because students shared nutrition breaks between activities. To our knowledge, previous studies have not reported on the importance of including unstructured social opportunities when setting up ISL experiences. These were critical experiences that fostered team work and camaraderie. The overarching theme of safety and comfort was also different from the typical stress encountered in most uni-disciplinary simulation activities (Parker \& Myrick, 2011).

\section{Study limitations}

The main limitation of this study is related to sample size. Although this was a qualitative study and we reached data saturation, we still wonder if a larger group of participants could have generated different results, including participants who may not have found the ISL activities valuable. A second limitation involved the voluntary nature of the participation in the study. If ISL activities were required in our program rather than voluntary - as in this study, students may have different perceptions about the value of ISL experiences. 


\section{Conclusion}

\section{Implications for curriculum development and further research}

The positive outcomes that resulted from the ISL activities could have significant implications for curriculum development and program delivery. Participants suggested that this type of ISL was of value but recognized that curricula are already heavy; thus, inclusion of such ISL should be done strategically and in a way that does not increase faculty or student workload. More importantly, the study successfully used existing scenarios from two professions to create an ISL experience. This implies that readily available components of curricula can be easily modified into interprofessional learning experiences, thus saving time and resources-a notable consideration in the delivery of such modules (Efsathkiou \& Walker, 2013) and in the context of fiscal restraint. Student passion and engagement was essential to project success and was facilitated by the voluntary nature of participation. Steps would need to be taken to ensure similar enthusiasm in curricular ISL activities; this may include removing any evaluation to promote student comfort. The efficiency and impact of ISL can be profound. Some students compared the ISL used in this project to other interdisciplinary learning opportunities, noting that the ISL was more memorable than classroom activities they had previously experienced. Furthermore, they emphasized the safety associated with having an encounter with another profession in a simulated learning environment as opposed to a clinical setting.

The timing of ISL is an important consideration on which there was no consensus. Some students suggested that ISL be introduced early in the program to encourage early interprofessional relationship building; others favoured including ISL toward the end of their programs, as their professional maturity would enhance the interprofessional learning. This study also included only two professions; adding more opportunities for encounters with other professions would add value to student learning but might be unmanageable when considering timetabling issues. Based on these findings and further reflection, we suggest a series of ISL opportunities where students encounter different professions over time. With respect to the structure of ISL activities, we remark that interprofessional faculty facilitation is critical-particularly during debriefing-to promote reflection and address misperceptions or assumptions. For example, faculty can facilitate discussion about perceived differences in professional language and philosophy of care that in fact may not be as different as they seem (e.g., patient-centred, holistic) and can offer additional perspective arising from their broad experience as seasoned professionals. Faculty can also foster collaborative thinking about what unites health professionals. By focusing on shared values, students of each profession can reflect on how they can best work together. For ISL activities conducted across professions, a critical evaluation of the process and outcomes can build a body of evidence for best teaching practices.

\section{Summary}

Results from this study lend support to using ISL activities to promote interprofessional teamwork and learning about one's own and another profession. It also provides a foundation for further study of the optimum structure and timing of such ISL activities within nursing and other health science curricula. 


\section{References}

Ateah, C., Snow, W., Wener, P., Macdonald, L., Metge, C., Davis, P., ...Anderson, J. (2011). Stereotyping as a barrier to collaboration: Does interprofessional education make a difference? Nurse Education Today, 31, 208-213.

Baker, C., Pulling, C., McGraw, R., Dagnone, J.D., Hopkins-Rosseel, D., \& Medves, J. (2008). Simulation in interprofessional education for patient-centred collaborative care. Journal of Advanced Nursing, 64(4), 372-379.

Bainbridge, L., Nasmith, L., Orchard, C., \& Wood, V. (2010). Competencies for interprofessional collaboration. Journal of Physical Therapy Education, 24(1), 6-11.

Bandali, K., Parker, K., Mummery, M., \& Preece, M. (2008). Skills integration in a simulated interprofessional environment: An innovative undergraduate applied health curriculum. Journal of Interprofessional Care, 22(2), 179-189.

Dagnone, J.D., McGraw, R., Pulling, C., \& Patteson, A. (2008). Interprofessional resuscitation rounds: A teamwork approach to ACLS education. Medical Teacher, 30(2), e49-54.

Durning, S.J., \& Artino, A.R. (2011). Situativity theory: A perspective on how participants and the environment can interact: AMEE Guide no. 52. Medical Teacher, 33(3), 188-199.

Efstathiou, N., \& Walker, W.M. (2013). Interprofessional, simulation based training in end of life care communication: A pilot study. Journal of Interprofessional Care, Early Online, 1-3, posted online on August 21, 2013.

Fernandez, R., Parker, D., Kalus, J. S., Miller, D., \& Compton, S. (2007). Using a human patient simulation mannequin to teach interdisciplinary team skills to pharmacy students. American Journal of Pharmaceutical Education, 71(3), 51.

Field, P.A., \& Morse, J. (1985). Nursing Research: The Application of Qualitative Approaches. Rockville, MD: Aspen Publishers, Inc.

Graneheim, U.H., \& Lundman, B. (2003). Qualitative content analysis in nursing research: Concepts, procedures and measures to achieve trustworthiness. Nurse Education Today, 24, 105-112.

Gum, L., Greenhill, J., \& Dix, K. (2010). Clinical simulation in maternity (CSiM): Interprofessional learning through simulation team training. Quality and Safety in Health Care, 19(5), 1-5.

Hean, S., Clark, J., Adam, K., \& Humphris, D. (2006). Will opposites attract? Similarities and differences in students' perceptions of the stereotype profiles of other health and social care professional groups. Journal of Interprofessional Care, 20 (2), 162-181.

Health Canada (2006). Interprofessional education for collaborative patient-centred practice. Retrieved from http://www.hc-sc.gc.ca/hcs-sss/pubs/hhrhs/2006-iecps-fipccp-workatel/indexeng.php. 
MacDonnell, C.P., Derraza, A.J., Lavin, M.D., Cohen, S.A., \& Cohen, L. (2011). Impact of an interdisciplinary practice laboratory on pharmacy and nursing students' perceptions of health care roles. The International Journal of Pharmacy Education and Practice, 7(1).

Merién, A.E., van de Ven, J., Mol, B.W., Houterman, S., \& Oei, S.G. (2010). Multidisciplinary team training in a simulation setting for acute obstetric emergencies: A systematic review. Obstetrics and Gynecology, 115(5), 1021-1031.

Mezirow, J. (1994). Understanding transformative theory. Adult Education Quarterly, 44(4), 222-232.

Mezirow, J. (1995). Transformation theory of adult learning. In M. R. Welton (Ed.), In Defense of the Lifeworld (pp. 39-70). New York: SUNY Press.

O’Neill, B.J., \& Wyness, M.A. (2005). Student voices on an interprofessional course. Medical Teacher, 27(5), 433-438.

Parker, B.C., \& Myrick, F. (2010). Transformative learning as a context for human patient simulation. Journal of Nursing Education, 49(6), 326-332.

Robertson, J., \& Bandali, K. (2008). Bridging the gap: Enhancing interprofessional education using simulation. Journal of Interprofessional Care, 22(5), 499-508.

Roper, J.M., \& Shapira, J. (2000). Ethnography in Nursing Research. Thousand Oaks, CA: Sage.

Taylor, D.C.M., \& Hamdy, H. (2013). Adult learning theories: Implications for learning and teaching in medical education: AMEE Guide No. 83. Medical Teacher, 35(11), e1561-e1572.

Tinsley, R., \& Leback, K. (2009). Expanding the zone of reflective capacity: Taking separate journeys together. Networks: An Online Journal for Teacher Research, 11(2), 1-11.

Vaismoradi, M., Turunen, H., \& Bondas, T. (2013). Content analysis and thematic analysis: Implications for conducting a qualitative descriptive study. Nursing and Health Sciences, 15(5), 398-405.

Wells, G. (2000). Dialogic inquiry in education: Building on the legacy of Vygotsky. In C. D. Lee \& P. Smagorinsky (Eds.), Vygotskian Perspectives on Literacy Research: Constructing Meaning through Collaborative Inquiry. Cambridge, UK: Cambridge University Press.

Westberg, S.M., Adams, J., Thiede, K., Stratton, T.P., \& Bumgardner, M.A. (2006). An interprofessional activity using standardized patients. American Journal of Pharmacy Education, 70(2), N_0214-N_0218. 